05. 1;06.5;10

\title{
Диагностика остаточных напряжений в биметаллических пластинах алюминий-сталь с использованием лазерного ультразвука и зондирующего отверстия
}

\author{
(С) А.Л. Глазов, К.Л. Муратиков \\ Физико-технический институт им. А.Ф. Иоффре РАН, Санкт-Петербург, Россия \\ E-mail: glazov.holo@mail.ioffe.ru
}

Поступило в Редакцию 23 июня 2020г.

В окончательной редакции 18 июля 2020г.

Принято к публикации 20 августа 2020 г.

Исследован характер изменения лазерных фотоакустических сигналов вблизи отверстий малого диаметра в слоях алюминия и стали в биметаллической пластине, полученной методом горячей прокатки. Проведено сравнение экспериментальных результатов с решением классической задачи Кирша для распределения напряжений вокруг круглого отверстия в предварительно напряженном материале. Показана возможность оценки внутренних напряжений лазерным фотоакустическим методом в сочетании с методом сверления отверстий в промышленных биметаллических пластинах.

Ключевые слова: фотоакустическая микроскопия, механические напряжения, термоупругость, биметаллы, горячая прокатка.

DOI: 10.21883/PJTF.2020.23.50346.18435

Исследованию возможности использования лазерных ультразвуковых и фотоакустических (ФА) методов для диагностики внутренних напряжений в современных материалах уделяется все большее внимание [1,2]. Их важными достоинствами являются универсальность, неразрушающий характер и возможность контролировать не только поверхностные характеристики материалов, но и свойства подповерхностных слоев. К настоящему времени продемонстрирована чувствительность лазерных ФА-методов к механическим напряжениям в керамиках нитрида кремния [3] и трехкомпонентных керамиках $\mathrm{Al}_{2} \mathrm{O}_{3}-\mathrm{SiC}-\mathrm{TiC}$ [4], а также в металлах на примерах стали [5] и алюминия [6,7]. Во всех перечисленных работах исследования были выполнены на однородных модельных образцах, что является определенным сдерживающим фактором дальнейшего применения лазерных ФА-методов для диагностики остаточных напряжений. Вместе с тем считается, что применение лазерных ультразвуковых и ФА-методов может быть особенно эффективным для изделий со сложной реологией, производимых в том числе по новым аддитивным технологиям и с применением методов 3D-печати $[8,9]$.

В связи с этим целью настоящей работы является исследование внутренних напряжений лазерным ФА-методом в промышленных биметаллических пластинах алюминий-сталь, получаемых методом горячей прокатки и широко используемых в судостроительных корпусных конструкциях [10-12]. Исследования были проведены на пластинах типа КБ-М-1Т [13] общей толщиной $8 \mathrm{~mm}$, состоящих из $4 \mathrm{~mm}$ слоя алюминиевого сплава 1561 и $4 \mathrm{~mm}$ слоя стали Д40. Их соединение осуществлялось методом горячей прокатки с введением промежуточного слоя алюминиевого сплава марки АД1 толщиной $0.3 \mathrm{~mm}$.

Для оценки внутренних напряжений в композитных металлических пластинах чаще всего используется метод сверления отверстий в совокупности с тензорезистивными датчиками $[14,15]$. Однако этот метод, имея невысокое пространственное разрешение, позволяет проводить оценку остаточных напряжений только на поверхностях пластин. В настоящей работе предложен метод исследования распределения напряжений в поперечном сечении многослойной пластины с микронным разрешением. Предлагаемый метод основан на линейной зависимости ФА-сигнала от механических напряжений. При этом коэффициент пропорциональности зависит от внутренней структуры материала.

Ранее нами было показано, что для определения указанной линейной зависимости можно использовать метод сверления отверстий с последующим приложением внешней нагрузки [6,7]. Для исследуемого композитного материала сначала были проведены такие измерения на поверхностях алюминиевой и стальной частей. Методика эксперимента описана, например, в работе [16]. На рис. 1, а представлено ФА-изображение части поверхности образца вокруг отверстия диаметром $0.2 \mathrm{~mm}$ со стороны алюминиевого сплава в исходном состоянии. Размер изображения $1 \times 1 \mathrm{~mm}$. Изображения получены при двумерном сканировании поверхности лазерным пучком диаметром $20 \mu \mathrm{m}$ с шагом $5 \mu \mathrm{m}$. Сигнал регистрировался на резонансной частоте $101.5 \mathrm{kHz}$ с цилиндрического пьезодатчика, прикрепленного к тыльной стороне образца. Характерное распределение сигнала вокруг отверстия свидетельствует о наличии однородных остаточных напряжений в поверхностном слое. Для 

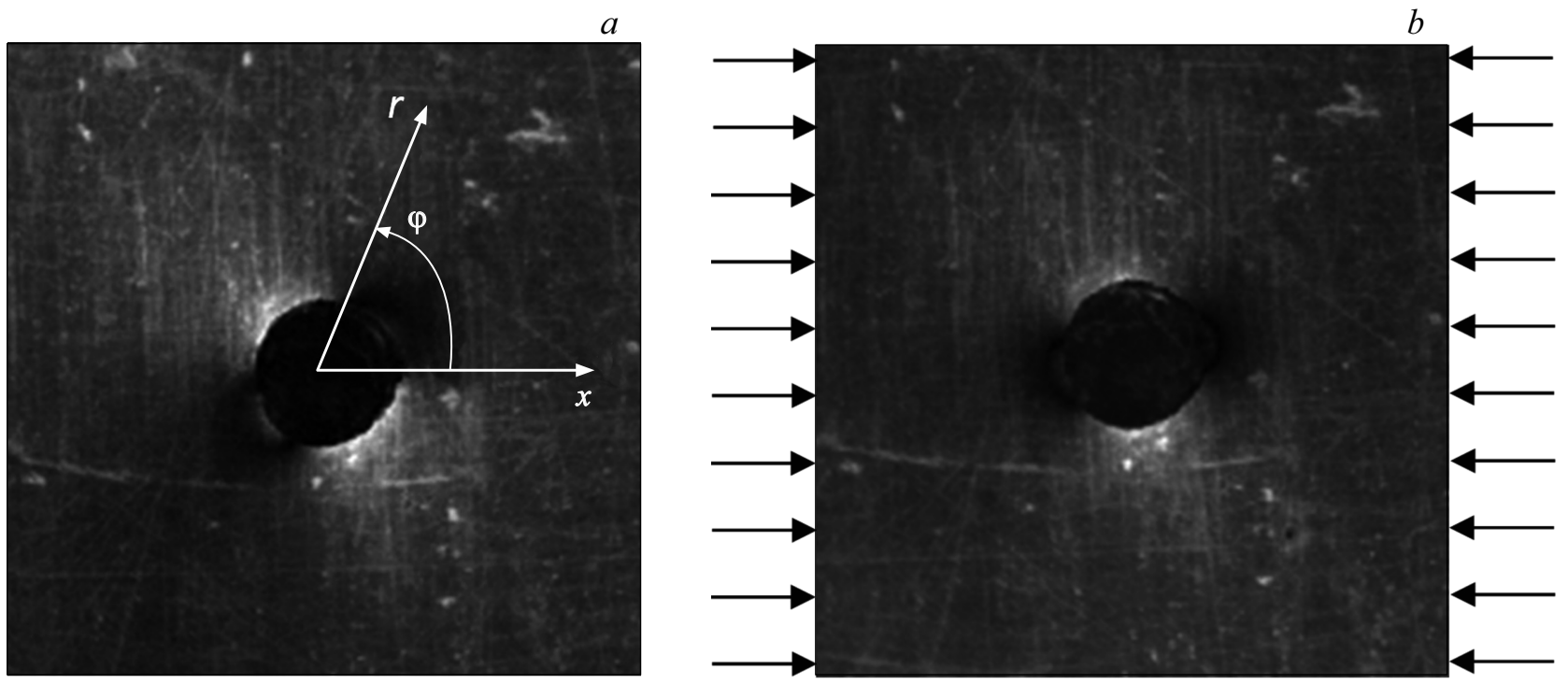

Рис. 1. ФА-изображения поверхности композитного металлического образца со стороны алюминиевого сплава. $a-$ образец в начальном состоянии, $b$ - образец под сжимающей одноосной нагрузкой $63 \mathrm{MPa} \mathrm{(направление} \mathrm{приложенного} \mathrm{давления} \mathrm{обозначено}$ стрелками). Размер изображений $1 \times 1 \mathrm{~mm}$.

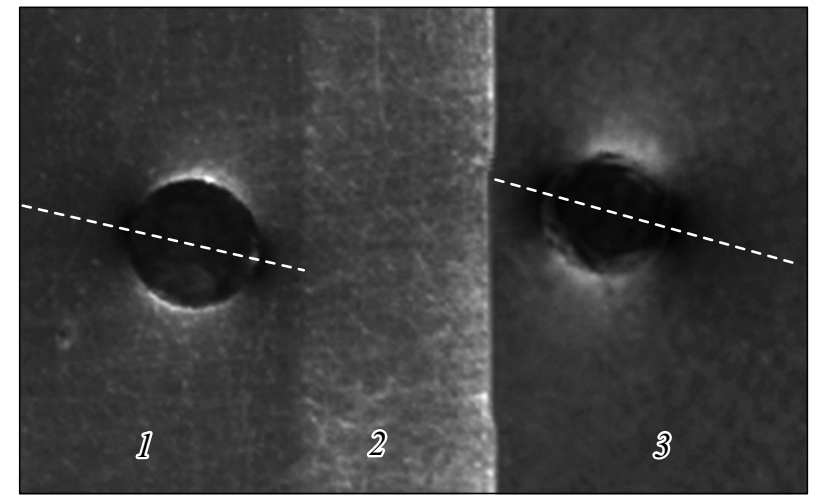

Рис. 2. ФА-изображения композитного металлического образца в плоскости, перпендикулярной границе слоев. 1 - алюминиевый сплав 1561, 2 - промежуточный слой из алюминиевого сплава АД1, 3 - сталь Д40. Размер изображения $1.3 \times 0.8 \mathrm{~mm}$.

оценки этих напряжений было приложено давление в направлении оси $x$, показанном на рис. 1. ФА-сигналы без нагрузки и под нагрузкой $63 \pm 3 \mathrm{MPa}$ хорошо аппроксимируются функцией [6,7]:

$$
S=S_{\infty}\left(1+A \cos \left(2 \varphi-2 \varphi_{0}\right) / r^{2}\right)
$$

с соответствующими подгоночными параметрами $S_{\infty}, A$ и $\varphi_{0}$, где $S_{\infty}-$ сигнал вдали от отверстия, $\varphi_{0}-$ угол между одной из главных осей напряжения и осью $x$. Для количественной интерпретации сигнала и оценки напряжений, изначально присутствующих в данном образце, рассмотрим следующую задачу. Пусть изначально в пластине присутствуют остаточные одноосные напряжения $\sigma_{1}$ и $\sigma_{2}$, параллельные поверхности пластины и действующие под углами $\alpha$ и $\alpha+\pi / 2$ к оси $x$. Тогда распределение суммарного напряжения $\sigma=\sigma_{r r}+\sigma_{\theta \theta}$ вокруг отверстия в полярных координатах [17]:

$$
\sigma=\sigma_{1}+\sigma_{2}-2 a^{2}\left(\sigma_{1}-\sigma_{2}\right) \cos (2 \varphi-2 \alpha) / r^{2},
$$

где $a$ - радиус отверстия. При приложении нагрузки $\sigma_{x}$ вдоль оси $x$ добавляется дополнительное напряжение $\sigma^{\prime}=\sigma_{x}\left(1-2 a^{2} \cos (2 \varphi) / r^{2}\right)$. Складывая оба этих напряжения, получим

$$
\sigma^{\prime \prime}=\sigma_{1}+\sigma_{2}+\sigma_{x}-B \cos (2 \varphi-2 \beta) / r^{2},
$$

где

$$
\begin{gathered}
B=2 a^{2} \sqrt{\sigma_{x}^{2}+\left(\sigma_{1}-\sigma_{2}\right)^{2}+2 \sigma_{x}\left(\sigma_{1}-\sigma_{2}\right) \cos (2 \alpha)}, \\
\operatorname{tg}(2 \beta)=\left(\sigma_{1}-\sigma_{2}\right) \sin (2 \alpha) /\left(\sigma_{x}+\left(\sigma_{1}-\sigma_{2}\right) \cos (2 \alpha)\right) .
\end{gathered}
$$

Из последнего выражения легко получить

$$
\sigma_{1}-\sigma_{2}=\sigma_{x} \sin (2 \beta) / \sin (2 \alpha-2 \beta) .
$$

Таким образом, найдя углы поворота осей симметрии на рис. $1, a$ и $b$, можно оценить остаточное напряжение в образце без внешней нагрузки.

Для представленных на рис. 1 изображений, осуществляя подгонку трех параметров методом наименьших квадратов, получим следующие значения углов поворота одной из главных осей напряжений относительно оси $x$ : для ненагруженного состояния $\alpha=0.670 \pm 0.002 \mathrm{rad}$ и для нагруженного состояния $\beta=0.227 \pm 0.002 \mathrm{rad}$. Тогда, согласно формуле $(6), \sigma_{1}-\sigma_{2}=(0.566 \pm 0.008) \sigma_{x}=-40 \pm 4 \mathrm{MPa}$, где знак минус означает, что к образцу прикладывалась сжимающая нагрузка $\sigma_{x}<0$. 

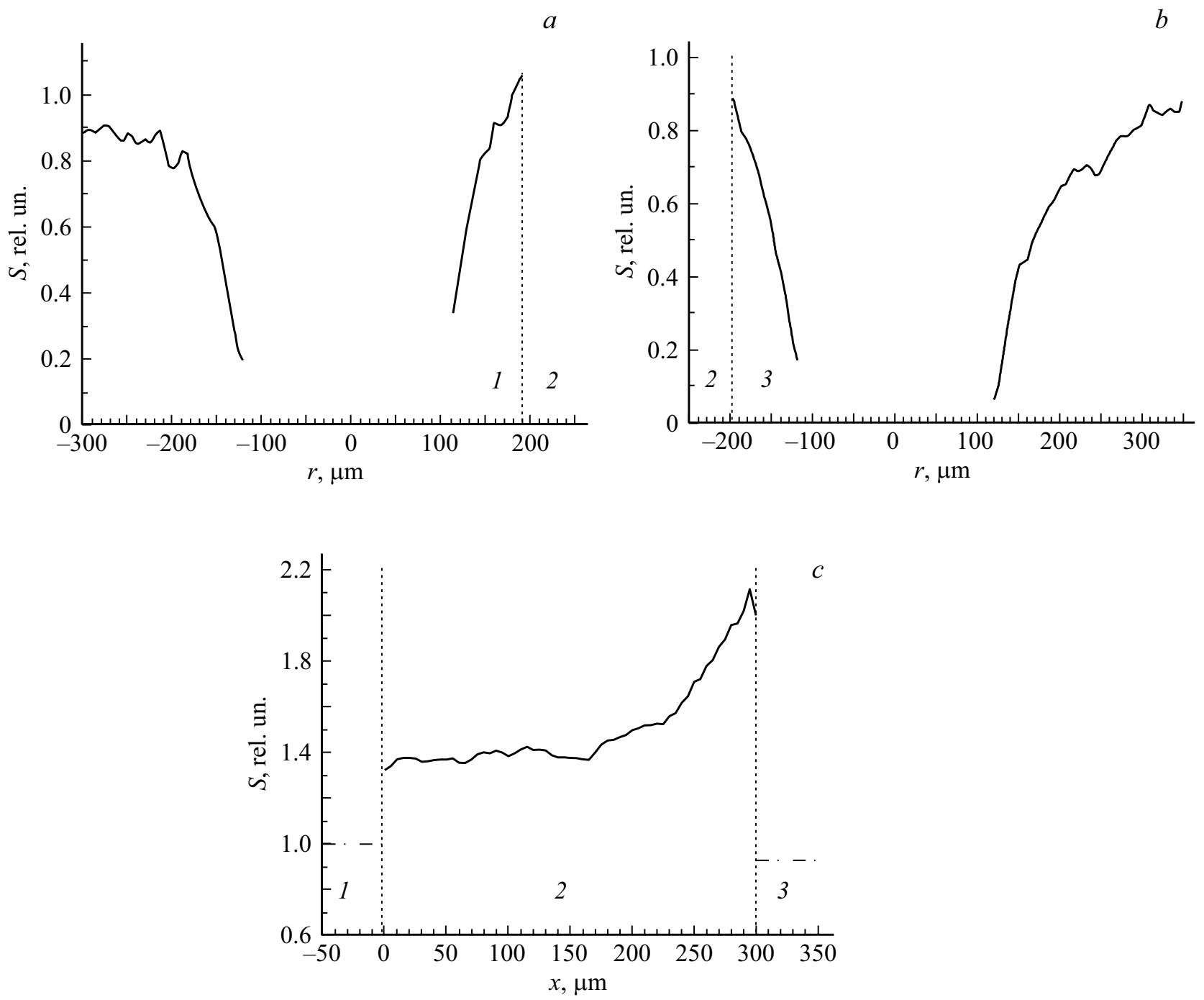

Рис. 3. $a-$ ФА-сигнал около отверстия в слое Al 1561 вдоль штриховой линии, показанной на рис. 2 ; $b-$ то же для стального слоя; $c$ - усредненный вдоль границы слоев ФА-сигнал поперек промежуточного слоя. 1 - слой $\mathrm{Al} 1561,2-$ промежуточный алюминиевый слой, 3 - стальной слой. Вертикальные штриховые линии - границы слоя. Штрихпунктирные линии - средние значения ФА-сигнала вдали от отверстий в алюминиевой и стальной пластинах.

Для определения коэффициента $b$ в линейной зависимости ФА-сигнала от напряжения для данного материала подставим в формулу $S=S_{0}(1+b \sigma)$ выражение для напряжений (2). Сравнивая полученный результат с распределением сигнала (1), нетрудно выразить коэффициент $b$ через подгоночный параметр $A$ :

$$
b=-A /\left(A\left(\sigma_{1}+\sigma_{2}\right)+2 a^{2}\left(\sigma_{1}-\sigma_{2}\right)\right) .
$$

Таким образом, для нахождения $b$ необходимо знать оба напряжения $\left(\sigma_{1}\right.$ и $\left.\sigma_{2}\right)$. Предположим, что напряжение в пластине одноосное, т.е. либо $\sigma_{1}=0$, либо $\sigma_{2}=0$, причем известно, что в композитных пластинах, сваренных под давлением, имеется растягивающее остаточное напряжение $[14,18,19]$. В этом случае $\sigma_{1}=0$ и в приповерхностном слое пластины из алюминиевого сплава имеется остаточное растягивающее напряжение $\sigma_{2}=40 \pm 4 \mathrm{MPa}$. Для ФА-изображения на рис. $1, a$ под- гоночный параметр $A=-25900 \pm 100 \mu \mathrm{m}^{2}$. Тогда коэффициент $b=-0.014 \pm 0.003 \mathrm{MPa}^{-1}$ для обоих изображений. Следует отметить, что такой же коэффициент был получен для алюминиевого сплава Д16 в работе [6].

Аналогичные исследования были проведены для стальной поверхности. При этом было установлено наличие остаточного растягивающего напряжения $80 \pm 10 \mathrm{MPa}$. Коэффициент $b \approx-0.005 \pm 0.001 \mathrm{MPa}^{-1}$.

Для исследований остаточных напряжений в поперечном сечении образца были получены ФА-изображения поверхности поперечного сечения. На рис. 2 представлено ФА-изображение поперечного сечения с двумя отверстиями в алюминиевом сплаве и стали около интерфейса. Ось $z$ на этом графике направлена перпендикулярно свободной поверхности слоя из алюминиевого сплава. На изображении также виден промежуточный слой из алюминия. Характерное распределение 
сигнала вокруг отверстий с учетом полученных выше результатов указывает на наличие растягивающих напряжений вдоль границы в алюминиевой $(21 \pm 6 \mathrm{MPa})$ и стальной $(70 \pm 20 \mathrm{MPa})$ пластинах непосредственно около промежуточного слоя. Численные значения были получены с использованием определенных выше структурных коэффициентов $b$ и обращенной формулы (7). Следует отметить, что, так как отверстия находились близко к границе промежуточного слоя, напряжения нельзя считать строго однородными. Однако оценки влияния свободной границы на деформации в такой геометрии показывают, что в точке на окружности отверстия, максимально удаленной от края полуплоскости, даже при расстоянии от центра отверстия до края 1.3 радиуса напряжение отличается не более чем на $10 \%$ от значений для удаленного отверстия [17]. В случае границы между двумя металлами эта разница значительно меньше. На рис. 3, $a$ и $b$ показаны сечения изображения, представленного на рис. 2, около отверстий в алюминиевом и стальном слоях вдоль одной из главных осей напряжений, показанных на рис. 2 штриховой линией. На обоих сечениях видно влияние промежуточного слоя. Для уменьшения этого влияния при аппроксимации использовалась только дальняя от границы промежуточного слоя половина изображения вокруг отверстия.

Интересно проследить за поведением сигнала внутри промежуточного слоя из алюминиевого сплава АД1. Усредненное вдоль слоя распределение ФА-сигнала поперек этого слоя показано на рис. 3, c. Внутри слоя наблюдается большое увеличение ФА-сигнала. Поскольку коэффициент $b<0$, первый инвариант тензора напряжений отрицателен, что свидетельствует о быстром росте сжимающих напряжений к границе стального слоя. Этот результат согласуется с имеющимися литературными данными $[19,20]$.

Полученные результаты измерения остаточных напряжений хорошо согласуются с известными распределениями напряжений в композитных металлических пластинах, формируемых методом горячей прокатки. Предложенный подход позволяет оценивать остаточные напряжения в различных слоях таких материалов с высокой степенью локальности. Используя получаемые данные о зависимости ФА-сигнала от напряжений для определенного материала, можно оценивать напряжения в областях вдали от пробного отверстия.

\section{Благодарности}

Авторы выражают благодарность НИЦ „Курчатовский институт“ и ЦНИИ КМ „Прометей“ за предоставление образцов биметаллических пластин, а также В.И. Павловой за обсуждение полученных результатов.

\section{Конфликт интересов}

Авторы заявляют, что у них нет конфликта интересов.

\section{Список литературы}

[1] Huan H., Lin L., Mandelis A., Peng C., Chen X., Zhan J. // Appl. Sci. 2020. V. 10. P. 1588 (1-16). https://doi.org/10.3390/app10051588

[2] Huan H., Mandelis A., Lashkari B., Liu L. // Int. J. Thermophys. 2017. V. 38. P. 62 (1-11). https://doi.org/10.1007/s10765-017-2197-7

[3] Muratikov K.L., Glazov A.L., Rose D.N., Dumar J.E. // J. Appl. Phys. 2000. V. 88. P. 2948-2955. https://doi.org/10.1063/1.1287526

[4] Муратиков К.Л., Глазов А.Л. // ЖТФ. 2003. Т. 73. В. 8. C. $90-97$.

[5] Глазов А.Л., Морозов Н.Ф., Муратиков К.Л. // ФТТ. 2016. T. 58. B. 9. C. $1679-1687$.

[6] Глазов А.Л., Муратиков К.Л. // Письма в ЖТФ. 2019. Т. 45. B. 17. C. 51-54. DOI: 10.21883/PJTF.2019.17.48226.17892

[7] Глазов А.Л., Морозов Н.Ф., Муратиков К.Л. // ДАН. 2019. T. 484. № 3. C. $277-280$. https://doi.org/10.31857/S0869-56524843277-280

[8] Millon C., Vanhoye A., Obaton A.F., Penot J.D. // Weld. World. 2018. V. 62. P. 653-661. https://doi.org/10.1007/s40194-018-0567-9

[9] Yu J., Zhang D., Li H., Song C., Zhou X., Shen S., Zhang G., Yang Y., Wang H. // Appl. Sci. 2020. V. 10. P. 365 (1-12). https://doi.org/10.3390/app10010365

[10] An J., Liu Y., Lu Y., Sun D. // Mater. Charact. 2001. V. 47. P. 291-297. https://doi.org/10.1016/S1044-5803(01)00190-5

[11] Yin F.X., Li L., Tanaka Y., Kishimoto S., Nagai K. // Mater. Sci. Technol. 2012. V. 28. P. 783-787. https://doi.org/10.1179/1743284711Y.0000000116

[12] Liu B.X., An Q., Yin F.X., Wang S., Chen C.X. // J. Mater. Sci. 2019. V. 54. P. $11357-11377$. https://doi.org/10.1007/s10853019-03581-х

[13] Орыщенко А.С., Осокин Е.П., Павлова В.И., Зыков С.А. // Автоматическая сварка. 2009. № 10. С. 43-47. https:/patonpublishinghouse.com/as/pdf/2009/as200910all.pdf

[14] Karolczuk A., Kowalski M., Kluger K., Źok F. // Arch. Metall. Mater. 2014. V. 59. P. 1129-1133. https://doi.org/10.2478/amm-2014-0195

[15] Karolczuk A., Paul H., Szulc Z., Kluger K., Najwer M., Kwiatkowski G. // J. Mater. Eng. Perform. 2018. V. 27. P. 4571-4581. https://doi.org/10.1007/s11665-018-3559-4

[16] Глазов А.Л., Морозов Н.Ф., Муратиков К.Л. // Физ. мезомеханика. 2019. Т. 22. № 2. С. 49-58. https://doi.org /10.24411/1683-805X-2019-12004

[17] Тимошенко С.П., Гудьер Джс. Теория упругости. М.: Наука, 1979. $560 \mathrm{c.}$

[18] Bonarski J.T., Kania B., Bolanowski K., Karolczuk A. // Arch. Metall. Mater. 2015. V. 60. P. 2247-2253. https://doi.org/10.1515/amm-2015-0370

[19] Sedighi M., Honarpisheh M. // Mater. Design. 2012. V. 37. P. 577-581. https://doi.org/10.1016/j.matdes.2011.10.022

[20] Yasheng $W$., Hongneng C., Ninxu M. // Strain. 1999. V. 35. P. 7-10. https://doi.org/10.1111/j.1475-1305.1999.tb01112 